\title{
Effective Venue Image Retrieval using Robust Feature Extraction and Model Constrained Matching for Mobile Robot Localization
}

\author{
Yue Feng ${ }^{1}$, Jinchang Ren ${ }^{2 *}$, Jianmin Jiang ${ }^{3}$, Martin Halvey ${ }^{4}$ and Joemon M. Jose ${ }^{5}$ \\ ${ }^{1}$ Department of Forensic and Neurodevelopmental Science, Institute of Psychiatry, King's College London, London, UK \\ ${ }^{2}$ Centre for excellence in Signal and Image Processing, EEE Dept., University of Strathclyde, Glasgow, UK \\ ${ }^{3}$ Digital Imaging and Systems Research Institute, University of Bradford, Bradford, UK \\ ${ }^{4}$ Interactive System Group, University of Glasgow, Glasgow, UK \\ ${ }^{5}$ Multimedia Information Retrieval Group, University of Glasgow, Glasgow, UK \\ yue.feng@kcl.ac.uk, jinchang.ren@strath.ac.uk, j.jiang1@bradford.ac.uk and \{halvey, jj\}@dcs.gla.ac.uk
}

\begin{abstract}
This paper describes a novel system for mobile robot localization in an indoor environment, using concepts like homography and matching borrowed from the context of stereo and content-based image retrieval techniques (CBIR). To deal with variations with respect to viewpoint and camera positions, a group of points of interest (POI) is extracted to represent the image for robust matching. To cope with illumination changes, we propose to produce a contrast image for each video frame by using the root mean square strategy, thus all the POIs are extracted from the corresponding contrast images to provide perceptually consistent measurement of image content. To achieve effective image matching, modeling of robot behavior for model constrained matching is proposed, where normalized cross correlation is employed for local matching to determine corresponding POI pairs followed by homography based global optimization using RANSAC. Meanwhile, application of specific constraints also helps to exclude irrelevant frames in the training set to further improve the efficiency and robustness. The proposed approach has been successfully applied to the Robot Vision task for the ImageCLEF workshop, and the experimental results have fully demonstrated the high-quality performance of our approaches in terms of both precision and robustness. The system and approach outlined in this paper was ranked the second best in the optional task group in ImageCLEF 2009. In addition to demonstrating the merits of our approach in isolation, we also illustrate the benefits of our proposed approach in comparison with other submissions.
\end{abstract}

Index Terms: content-based image retrieval, robot localization, computer vision, model constrained matching

*Corresponding Author:

Dr. Jinchang Ren

Centre of excellence in Signal and Image Processing

Department of Electronic and Electrical Engineering

University of Strathclyde

204 George Street

Glasgow, G1 1XW

United Kingdom

Tel. +44-141-5482384

Email. jinchang.ren@strath.ac.uk 


\section{INTRODUCTION}

Robot vision has a wide range of applications in both science and industries such as aerospace, security, and military. For example, in aerospace, the UAV (Unmanned Aerial Vehicles) airplane relies on a global positioning system (GPS) and visual sequence captured during flying to navigate a route. This process requires the system to detect landmarks in order to determine the position of the aircraft. Another example is in military services, robots are often used to explore an area that is too dangerous for soldiers, i.e. in an effort to reduce casualties. These types of operations require the military robot to annotate its environment so that it can be located. Thus, the requirement for constant and precise knowledge of the location and motion of the mobile robot is extremely critical.

One of the key objectives in mobile robotics is to provide an autonomous mobile robot with the ability to take a role in various tasks under different environments. To achieve this, the robot needs to be able to recognize its location; this can be achieved through some sort of localization methodology and has led to an important topic and area of research, namely mobile robot localization. Mobile robot localization has been a topic of interest for many years [16] [17], which aims to determine a robot's position in a map or in a learned map. Given the initial robot position, local tracking is the problem of keeping track of that position over time. Global localization provides mobile robots with the capabilities to deal with initialization and recovery from occlusion [18]. Visual based localization using natural landmarks is highly desirable in comparison with sensor based localization. Sensors such as sonar sensors and range finders can suffer from problems due to reflections; this problem can be eliminated when using only visual information for localization.

This paper describes our recent research on vision based mobile robot localization, which formed the basis of our submission for the ImageCLEF 2009 Robot Vision task [1]. The goal of this task is to address the problem of topological localization of a mobile robot using visual information. Given a test sequence, a system/algorithm must be able to provide information about the location of the robot, where part of the relevant location information is available in the training set. Since the determined location of the robot can be regarded as a kind of annotation, the corresponding task is considered as an extension of image annotation and retrieval. Details of the task are discussed in Section II (B).

In order to provide a solution for the task outlined, we are proposing the following solution which attempts to address and overcome many of the difficulties involved. The primary novelty of this paper is a methodology for obtaining robust feature extraction for image representation together with model constrained matching scheme where the moving behavior of the robot is modeled to guide the whole process, based on the concepts like homography and matching borrowed from the context of stereo. First, a contrast image is produced for each video frame, which can provide a perceptually consistent measurement of image content in spite of illumination changes. Secondly, a group of POIs is extracted from the corresponding contrast image for feature representation of images, which is invariant to changes of viewpoint and camera positions. Thirdly, modeling robot 
behavior for model constrained matching of POIs is employed for both efficiency and robustness, where normalized cross correlation and RANSAC are respectively applied to determine corresponding POI pairs and to determine a homography between two sets of POI pairs. It is worth noting that the model specific constraints can help improve the matching performance in terms of efficiency, accuracy and robustness by excluding irrelevant samples in the training set.

The remainder of this paper is organized as follows. In Section II, we describe previous state of the art in the area of robot vision and outline the objectives of robot vision task in ImageCLEF 2009. In Section III, the basic POI detector and its improved version using our derived contrast image are presented. In Section IV, modeling of robot behavior for model-constrained matching is presented. Experimental results from ImageCLEF 2009 and discussions are presented in Section V, and finally some concluding remarks are drawn in Section VI.

\section{RELATED WORK}

In this section we describe the state of the art in the area of mobile robot localization, including general solutions for this field and specific requirements from ImageCLEF 2009. Since this is the first time a mobile robot vision task has been introduced in the ImageCLEF workshop for benchmarking, the corresponding details for the task and workshop are also presented.

\section{A. Previous Work in General Mobile Robot Localization}

Castellanos et al. [20] propose a method for landmark-based map acquisition and robot localization where the use of external mechanisms, such as a Charge-Coupled Device (CCD) camera and a 2-D laser rangefinder, are implemented. In the cases addressed in this work, the robot followed a predefined trajectory and stopped at regular intervals to obtain information. Complementary information was taken, by hand, which provided real locations of the robot with respect to a base reference. A mapping algorithm with continuous localization has been proposed by Yamauchi et al. [21]. Sonar's, as well as laser rangefinders, were used with a greater confidence for the laser range data. The mapping was based on a certain exploration method, called Frontier-Based exploration. The algorithms outlined are based on sensor models and configuration, and on how detectable the selected features are. In the numerous cases where the sensor system can be unreliable, due to for example the ultrasonic transducers or because of effects like specular reflections or cross-talk, these modeling methods have additional problems. Moreover, these procedures are highly task dependent, so their parameters need to be re-adjusted to the conditions of the specific environment under exploration.

Recently, more attention has been focused on vision based methodologies for robot localization, which has mostly been adopted in robot navigation [2] and location recognition [3]. The main difficulty for vision based localization of robots is how to learn and determine the views of an environment with respect to viewpoint changes, which can result in different illuminations and occlusions for example. Most existing visual features are affected by varying viewpoints and illumination changes, thus 
these factors are extremely critical for an effective system.

The most popular vision based methods can be categorized into landmark recognition, geometric recognition, object recognition and probabilistic approaches. A great deal of previous work in this area relates to landmark-based methods, these methods use local features to detect vertical lines or specific signs for controlling robot motion [7]. The types of systems that use this approach usually require an a priori map of the environment. For example, Sim and Dudeck [22] use regions of the scene and images with a high number of edges as natural landmarks. The main advantage of landmark-based methods is that they have a bounded cumulative error. Although these features are easy to extract, they are not always available in image sequences.

Geometric methods mostly rely on sparse features such as POI and straight edges to describe a given scene, followed by an image matching algorithm, in order to match visual features of query images with images in the database. For example, Althaus et al. [8] use line features like detected edges to determine corridors and doorways. In Ohya et al. [6], an expectation image is first rendered from training images as a model, and then the current location of the robot is determined by matching edges extracted from the model image and a query image, respectively. However, their results are sensitive to the number of landmarks in the image, plus it also relies on information from an ultrasonic sensor, thus it is not purely visual.

Localization by using object recognition techniques have proved to be a promising approach, as these methods utilize natural features. Se et al. [23] use scale-invariant visual marks to deal with mobile robot localization based on the local feature detector and the SIFT descriptor proposed by Lowe [24]. Wang et al. propose a strategy based on the Harris-Laplace interest point detector and the SIFT descriptor [25]. In this system, each location is represented by a set of interest points instead of using all pixels. The system then compares one frame to all frames in the database using a nearest neighbor search. Katsura et al. [26] developed a system for detecting location in an outdoor local setting; the system can obtain the location by matching areas of trees, sky, and buildings. However, the matching in their approach is severely affected by occlusions.

A probabilistic approach normally uses machine learning methods to learn the properties of different locations, and then classify the query image into one of the classes based on the distance or probability between this image and every image class. Torralba et al. [4] use global gist features [5] to train a Gaussian mixture model (GMM) representation for a set of locations. Pronobis et al. [9] propose a method for visual place recognition by measuring the confidence level of the classification output from a support vector machine (SVM), based on the distance between a query image and the average distance of training vectors from each location. Since training classes usually need some prior knowledge of the training examples, they may produce unsatisfactory results if there is a lack of training examples, such as the samples of unknown rooms. Moreover, this approach is expensive in terms of computation. For example, for one single run it could take up to 2.5 days to finish, as reported in [9]. In addition, the number of training classes will increase greatly due to the possible combinations of different rooms and different illuminations, which may in turn harm the accuracy and also reduce the efficiency of the approach. 
Thus far we have highlighted various approaches to solve the difficult challenges associated with robot vision. In the following subsection, we describe the difficulties, challenges and the details of the new robot vision task from ImageCLEF 2009.

\section{B. Robot Vision Task in ImageCLEF Workshop}

According to the official webpage, ImageCLEF is the cross-language image retrieval track run as part of the Cross Language Evaluation Forum (CLEF) campaign starting from 2003 [19]. In ImageCLEF 2009, a robot vision task for mobile robot localization was introduced, with the aim of retrieving robot images using visual information. Accordingly, a task of high priority is defined and participants are asked to provide information about the location of the robot separately for each test image when only some of the images from the test sequences are available or the sequences are scrambled. This corresponds to the problem of global topological localization, where continuity of temporal information is not required. Meanwhile, an optional task is also defined for robot localization, in which exploiting continuity of the sequences and relying on the test images acquired before the classified image is allowed. It should be noted that for the workshop that an algorithm would be considered invalid if future frame information is used to classify current ones. Since temporal information has been employed in our approach for refined matching, our work is in the optional task group.

The robot vision task at ImageCLEF 2009 provided users with a common dataset and test bed on which state of the art techniques on robot localization techniques can be evaluated and ranked. For all the participants, a group of training data consisting of image sequences recorded in an indoor environment at a given time is provided, which includes a five-room subsection (kitchen, printer area, one-person office, two-person office and corridor) under three different illumination conditions (sunny, cloudy and night). The challenge for the participants in the robot vision task is to build a system able to answer the question 'where am I?' (I'm in the kitchen, in the corridor, etc), i.e. to assign each test image to one of the five venues or to indicate that the image comes from an unknown room. Moreover, the systems were allowed to refrain from making a decision (e.g. in the case of lack of confidence). As the moving speed of the robot is assumed to be limited, this allows the image matching function to work on a predictable range of possible matches.

The floor plan of the environment and samples of different rooms under different illumination conditions are shown in Fig.1 and Fig.2, respectively. As shown in Fig. 1, the five locales have quite different layout and settings. Though they can easily identified by humans, provided the whole 'image' of the venue is seen. However, as shown in Fig. 2, this becomes very difficult when only a very small part of the venue can be observed by the robot, additional difficulties for the task include variations in illumination conditions and camera view point. Since the test images are acquired 6-20 months after the training sequence, such variations are unavoidable and also may introduce more difficulties as discussed below.

One additional difficulty is introduced due to the unpredicted content changes in the captured images, which include adding, removing, or relocating of human or non-human objects. The latter can be found as furniture like chairs, curtains, room 
decorators and small objects on the table, etc. Consequently, matching of images for robot localization needs to be insensitive to such changes.

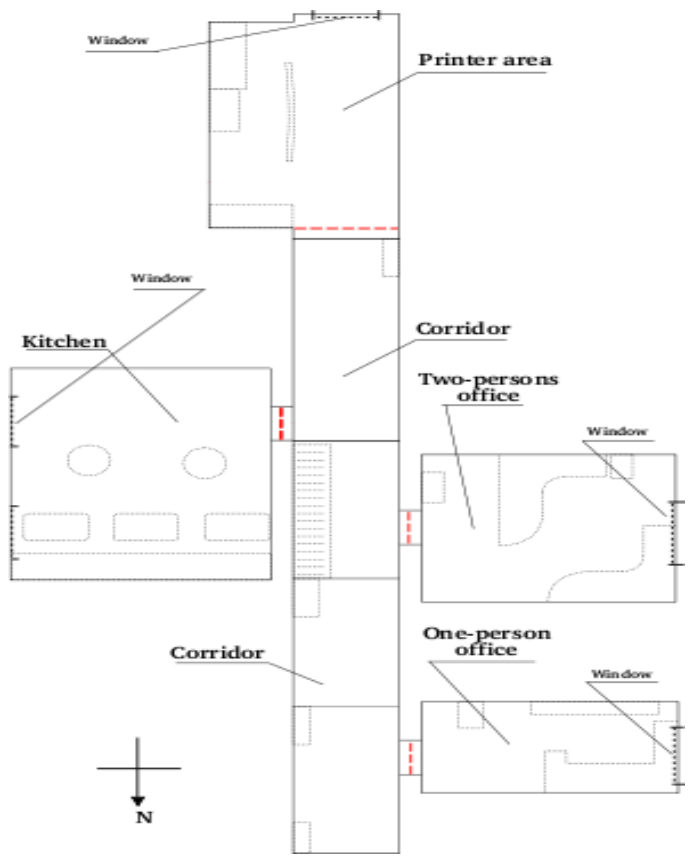

Fig 1. Map of an office environment.
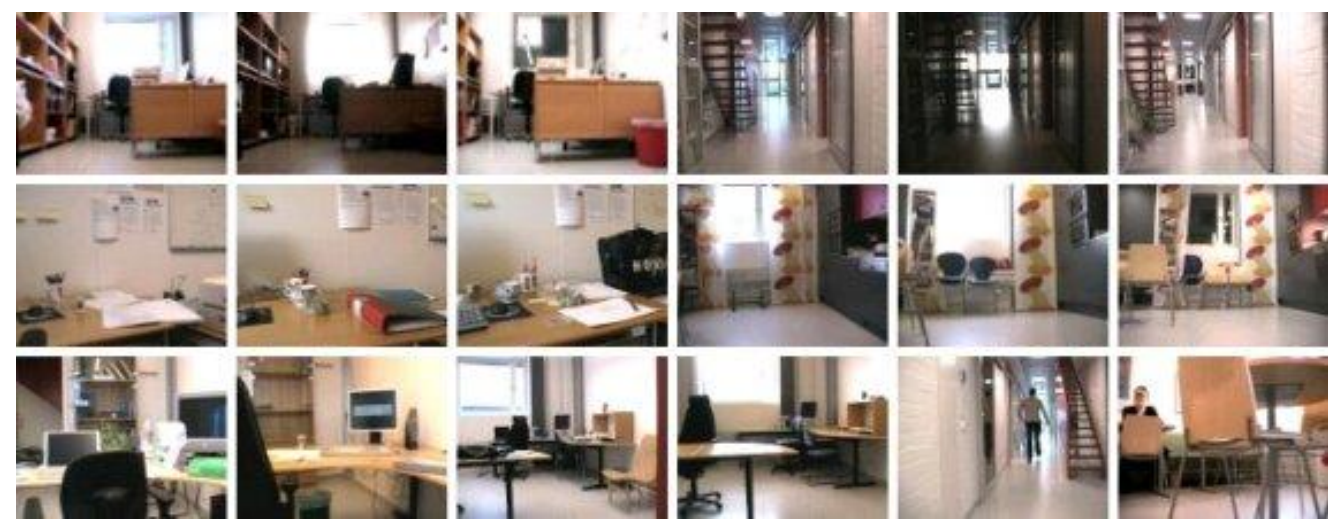

Fig 2. Sample frames in the training sequences. From top left to bottom right, the first 15 form 5 groups of images under 3 illumination conditions, which are captured from five venues including single-person office, corridor, printer area, kitchen, and two-person office, respectively. The last three images are twoperson office, corridor and kitchen, and they are used to show the content changes in comparison with other sample images within the same venue.

In addition, rooms which are excluded from training but used for testing also make the whole task more challenging. Since the office venues share common environmental and building conditions, as shown in Fig. 2, their images appear of quite similar background and comparable furniture settings. As a result, to locate an unknown room becomes an extremely hard problem for this task. 
To overcome these difficulties, we have proposed robust point based feature extraction and model constrained matching towards automatic robot localization in the robot vision task in ImageCLEF 2009. The system is mainly designed for use in autonomous navigation, where limited knowledge of the environment is available. The main assumption here is that the content information extracted from the scene provides enough features for matching. It is worth noting that this is achieved using vision based methods rather than external sensors, and the relevant details are discussed in the next two sections.

\section{ACCURATE AND RobUSt DETECTION OF POIS}

A key requirement for real-time robot location detection system is its fast performance on image matching. The matching problem that we have to solve, in order to find the images in the database that are most similar to the ones taken by the mobile robot, is one of so-called intensity-based image matching via full search [25]. This method captures the similarity between the intensity profiles of the two images. Given an intensity value at one pixel from one image, it searches looping through all the pixels on the other image to find the best match. This creates a problem of system efficiency, as for example pixels in the homogeneous texture domain contain high redundancy. Therefore, in our approach special attention is paid to selecting regions with higher information content instead of using all pixels.

Detection of POIs has recently received increasingly attention in the computer vision domain. An interest point is a point in the image that is rich in terms of local information content. In addition, an interest point is stable under local and global perturbations in the image domain, including deformations such as those arising from perspective transforms as well as illumination variations, such that the interest points can be reliably computed with high degree of reproducibility. Considering these characters, image matching via POI can be a good solution for our task for finding relevant images in image search, especially given its performance in illumination variations. In our approach, we have chosen corner features as POI since corners are discrete and partially invariant to scale and rotational changes.

\section{A. Original Harris Detector}

One of the most commonly used POI detection methods in computer vision applications is the Harris corner detector [10], which is used in our system as it is invariant to rotation, scale, illumination variation and image noise [38]. The Harris corner detector is based on Harris and Stephens improved upon Moravec's corner detector [11], by considering the differential of the corner score with respect to direction. This corner score is often referred to as autocorrelation, which can be described as follows. 


$$
\begin{aligned}
A(I, w) & =\left[\begin{array}{cc}
\sum_{(x, y) \in w}\left(\frac{\partial I(x, y)}{\partial x}\right)^{2} & \sum_{(x, y) \in w} \frac{\partial^{2} I(x, y)}{\partial x \partial y} \\
\sum_{(x, y) \in w} \frac{\partial^{2} I(x, y)}{\partial x \partial y} & \sum_{(x, y) \in w}\left(\frac{\partial I(x, y)}{\partial y}\right)^{2}
\end{array}\right] \\
& =\left[\begin{array}{cc}
\left\langle I_{x}^{2}\right\rangle & \left\langle I_{x} I_{y}\right\rangle \\
\left\langle I_{x} I_{y}\right\rangle & \left\langle I_{y}^{2}\right\rangle
\end{array}\right]
\end{aligned}
$$

where an image is given by $\mathrm{I} ; w$ represents an image patch over the area; $(\mathrm{x}, \mathrm{y})$ is a set of pixels around; and the angle brackets denote an averaging process.

The "cornerness" response function of the Harris corner detector is based on the determinant and trace of the autocorrelation matrix, where $k \in[0.04,0.15]$ as suggested in the literature [11].

$$
M_{\text {harris }}=\operatorname{det}(A)-k(\operatorname{trace}(A))^{2}
$$

\section{B. Dealing with Illumination Changes}

Although the conventional interest point detectors (IPD) can successfully locate corner points in images, generally they will fail or show lack of robustness when there are large illumination changes. To cope with the illumination changes in images, we detect points of interest from a normalized image, rather than the original image. Let $\varphi(\cdot)$ be a normalization function, the improved detector $M_{\text {prop }}$ is defined in Eq. (3) below, where $B$ is the autocorrelation matrix of the normalized image and $\hat{I}=\varphi(I)$ is the image of normalized contrast.

$$
\begin{aligned}
& M_{\text {prop }}=\operatorname{det}(B)-k(\operatorname{trace}(B))^{2} \\
& B(I, w, \varphi)=A(\hat{I}, w)=\left[\begin{array}{cc}
\left\langle\hat{I}_{x}^{2}\right\rangle & \left\langle\hat{I}_{x} \hat{I}_{y}\right\rangle \\
\left\langle\hat{I}_{x} \hat{I}_{y}\right\rangle & \left\langle\hat{I}_{y}^{2}\right\rangle
\end{array}\right]
\end{aligned}
$$

As seen, the normalization function $\varphi(\cdot)$ is the key to the solution above, where several typical options can be summarized as follows. In [12], Gevrekci et al. reported an approach called illumination robust feature extraction transform (IRFET). The idea is to stretch the image contrast as a function of intensity, in order to span the space of possible photometric transformations and to help to simulate a particular illumination condition. The contrast function introduced in that paper is given by

$$
\varphi_{I R F E T}(I(x, y))=\frac{1}{1+e^{-\ell(I(x, y)-c)}}
$$

where $I(x, y)$ is the normalized intensity value, $c$ is the contrast center around which the contrast is stretched, and $\ell$ is a 
parameter to determine the slope of the sigmoid function. Since $\varphi_{\text {IRFET }}$ is obtained for $c \in[0,1]$ with a step size of 0.05 , the overall results are very sensitive to the selection of $c$ from its 200 candidate values. Unfortunately, for a given image the method to select a suitable $c$ is undetermined. As a result, for an image pair it may be required to match $200 \times 200$ times to achieve the best result in dealing with illumination changes.

To overcome this drawback, we have adopted a different strategy for dealing with illumination changes. According to the characteristics of human visual perception, the details that we can observe from an image depend on a local contrast ratio rather than the intensity and this can be applied in our system to yield normalized image of refined contrast. We have considered three typical contrast functions including Weber, Michelson, and Root Mean Square as will be discussed below.

Weber contrast function [12] focuses on the luminance of the pixel and the background luminance.

$$
\varphi_{\text {weber }}(I(x, y))=\frac{I(x, y)-I_{b}}{I_{b}}
$$

where $I(x, y)$ and $I_{b}$ represents the luminance of the pixel and the background luminance, respectively. This function is commonly used in cases where small features are present on a large uniform background, i.e. the average luminance is approximately equal to the background luminance. However, this seems unsuitable for our task, as we intend to enhance the contrast of the background for effective extraction of POIs, rather than enhance the contrast of the foreground objects.

Michelson contrast [13] is another commonly used method to deal with luminance adjusting, which is defined as:

$$
\varphi_{\text {Michelson }}(I(x, y))=\frac{I_{\max }-I_{\min }}{I_{\max }+I_{\min }}
$$

where $I_{\max }$ and $I_{\min }$ refer to the maximum and the minimum intensity in an image region or a whole image. Due to the native of the definition, this function is sensitive to noise, especially when there are isolated bright and/or dark pixels.

Root Mean Square (RMS) contrast is defined as the standard deviation of the pixel intensities below [30],

$$
\varphi_{R M S}(I(x, y))=\sqrt{\frac{1}{M N} \sum_{x=0}^{N-1} \sum_{y=0}^{M-1}(I(x, y)-\bar{I})^{2}}
$$

where $I(x, y)$ is the intensity value of an image region sized $M * N, \bar{I}$ is the corresponding average intensity which has been normalized to 1 . As can be seen, RMS is insensitive to isolated noisy pixels and also helps to enhance the contrast of background pixels, as the standard deviation used can naturally smooth such noise and reflect the level of intensity changes in a local neighborhood. Consequently, the resulting image can keep fine details and enable effective extraction of POIs for matching.

For a given image, the contrast images obtained using the three functions above are shown in Fig. 3 , where a $5 \times 5$ local window is utilized in determining the local contrast. As can be seen, Weber fails to keep fine details even for areas whose 
intensities differ significantly from neighboring ones, such as the ceiling lights, though it generates less noise. On the contrary, the result of Michelson contains a great deal of noise as a result of abrupt contrast changes. Finally but not surprisingly, the result from RMS appears to be the best among this group of results. This is due to two main reasons: The first is normalized average intensity to overcome the effect of illumination changes, and the second is the root-mean-square processing used to help constrain noise [30]. Consequently, RMS has been employed in our system to generate a normalized contrast image in dealing with illumination changes.
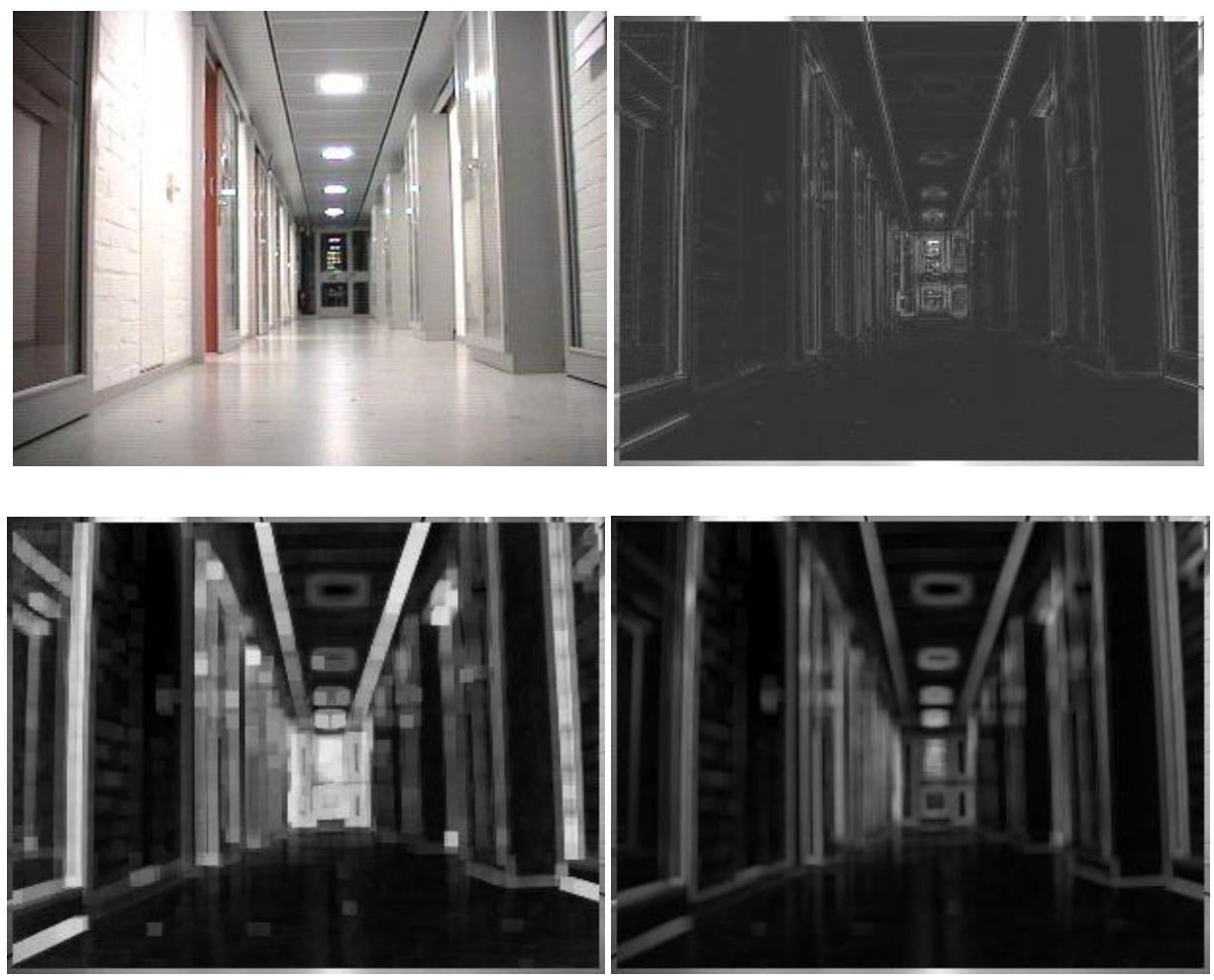

Fig 3. One original image (top left) and three contrast images obtained using functions of Weber (top right),

Michelson (bottom left) and RMS (bottom right), respectively.

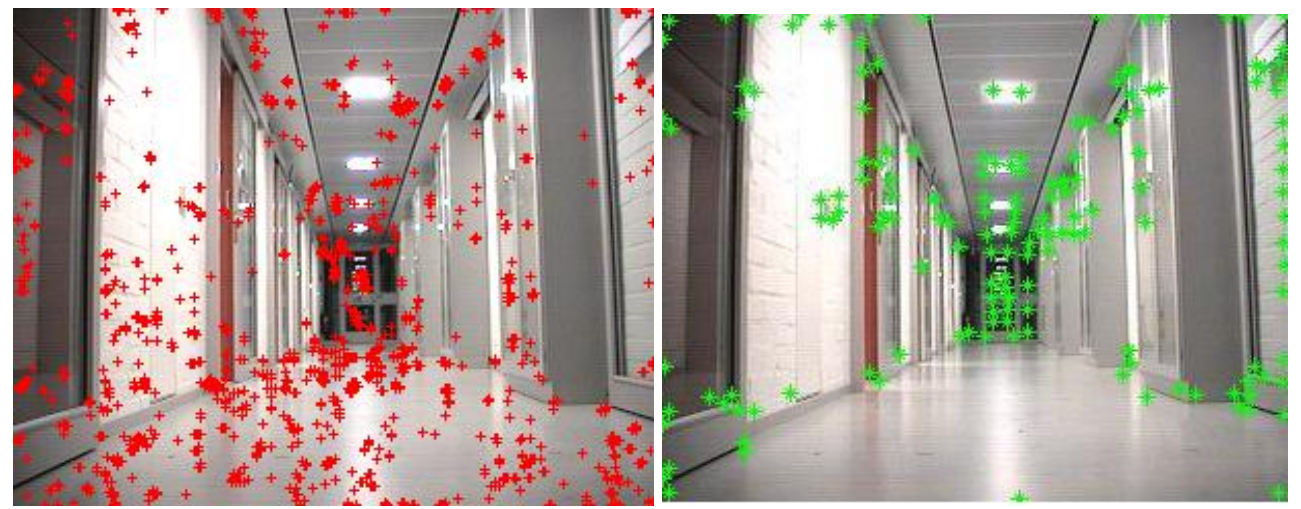

Fig 4. Extracted POIs from the original image (left) and the RMS contrast image (right) of Fig. 3. 
Based on the original image in Fig. 3, Fig. 4 shows results of extracted POIs using Harris detector (implemented in OpenCV), where the left and the right images are those extracted from the original image and the RMS contrast image, respectively. Unlike POIs extracted from the original image, POIs extracted from the RMS contrast image can reject those unreliable points on homogeneous regions like the floor whilst keeping useful points. Although RMS helps to reduce false corner points, it has detected much more corner points near the right boundary of the image and also one the door in the central area of the image. In other words, it helps to re-balance the distribution of the corner points in comparison to those detected from the original image. As a result, noise is suppressed towards more robust image matching in the next stage.

To provide further validation for our proposed strategy, we present another example of group of results of extracted POIs in Fig. 5, where the input image is under different illumination conditions. Again, most of unreliable POIs have been reduced whilst keeping useful points. These examples demonstrate the effectiveness of our introduced strategy in dealing with illumination changes. In the following section we outline our approach for introducing constraints on the model that we apply for robot localization.
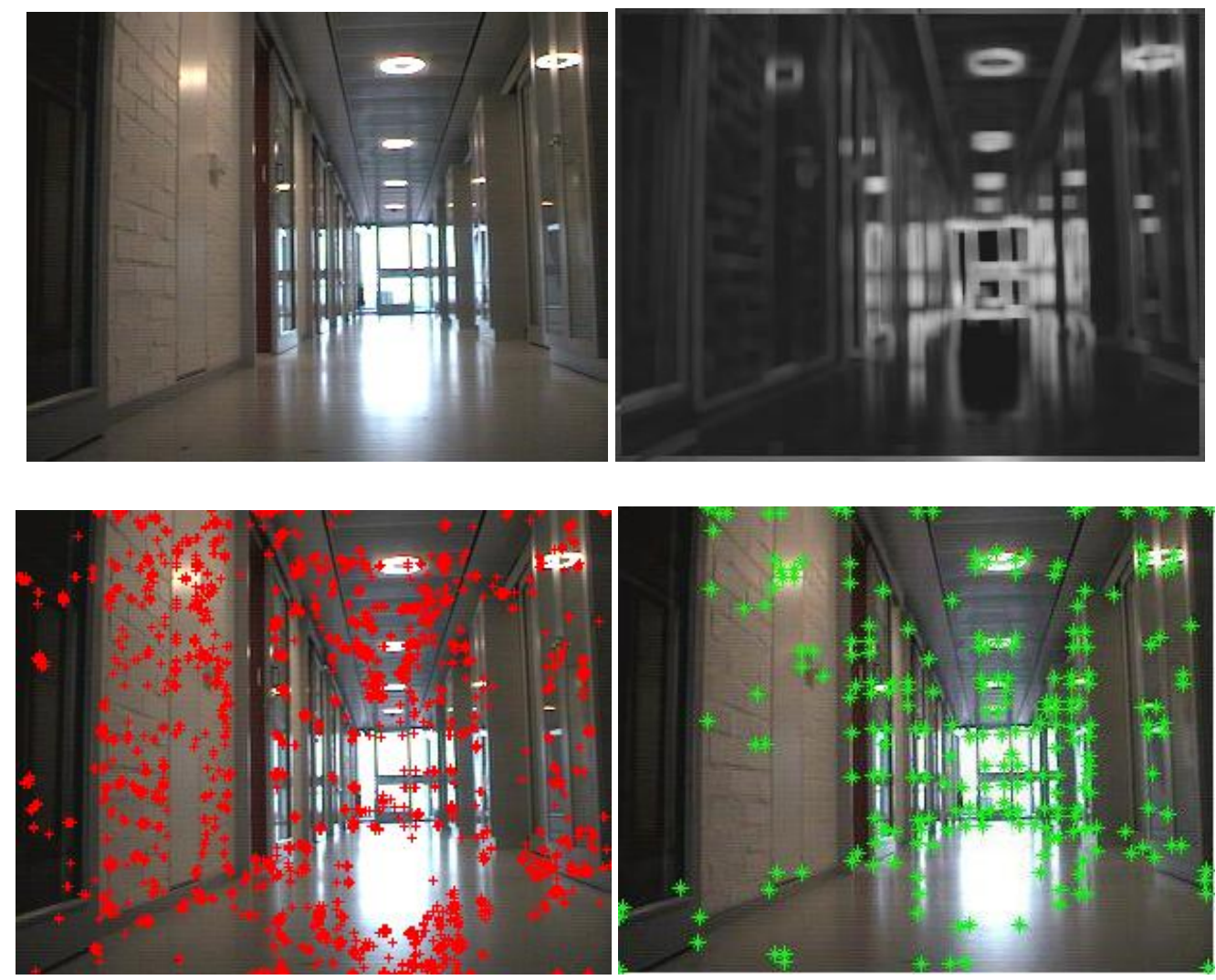

Fig 5. Another group of results under different illumination conditions: the top left one is the original image, and the top right one is its RMS contrast image. The bottom left and bottom right ones are extracted POIs from the original image and the RMS contrast image, respectively. 


\section{MODEL-CONSTRAINED MATCHING FOR RoBot LOCALIZATION}

Given the extracted POI in both training and test sequences, the next step is to compute the similarity between each test frame and all the frames in the training sequence to find the best match, so that the test frame will be annotated as the venue associated with the matched frame of the training set. To improve both efficiency and accuracy, application specific constraints are applied for matching the POIs. This strategy for feature matching and the relevant details for model-constrained frame annotation are outlined in the following subsections.

\section{A. Application Specific Modeling of Robot Behaviors}

By studying the environmental settings and the training sequences released as part of the Robot Vision task in ImageCLEF 2009, we find that the robot does not move 'randomly' and that the robot's behavior is somewhat constrained by environment settings. These constraints can be summarized into three rules as follows.

Rule 1: Time length. The period of time that the robot stays in one venue is always more than 3 seconds, i.e. 15 continuously frames at 5 fps. Accordingly, the robot will not stay in one place for a period less than $N_{0}$ frames, where $N_{0}$ is determined as being 20 as a result of statistically analyzing the training sequences.

Rule 2: Jumping room. The robot has to cross the corridor before it enters other venues such as kitchen, offices and printer area. In other words, the robot cannot bypass the corridor to go from one venue to another, i.e. jumping room is not allowed.

Rule 3: Unknown rooms. Since the test sequence contains additional rooms that are not included in the training sequences, no corresponding frames in the training set could be used to annotate these rooms. In addition, due to the nature of the image matching algorithm, that one test frame will be annotated with the most similar frame in the training set, a false annotation seems unavoidable. In our system, however, it was found that the consistency between the unknown frame and the training frames is very limited. This forms another rule for identifying unknown rooms, i.e. any test frame whose consistency below a given threshold will be annotated as an unknown room, details on how to define this consistency are outlined in Section IV $(\mathrm{C})$.

The rules described above have been generated by observing the data set and the behavior of the robot; it could be said that they are very specific for the robot vision task from ImageCLEF 2009. However, this type of methodology can be adapted and applied to other data sets for robot vision and localization, as in general the motion behavior of a robot is always constrained in a given closed environment. As the focus of this paper is on the application of the methodologies outlined above, we reserve the exploration of a more general solution for future work.

\section{B. Model-constrained Matching}

In order to facilitate the application of the three rules for robot localization outlined above, a model-constrained feature matching is employed in our system. When previous localization information is absent, e.g. for the first frame of the sequence, an 
exhaustive matching with all of the frames in the training set is required. Accordingly, the test frame will be classified into one of the 6 possible venues including printer area, corridor, two-person office, one-person office, kitchen or unknown room (applying Rule 3). Following on from this, the remaining two rules are applied as part of the matching process as follows.

If the robot is found within a venue for less than $N_{0}$ frames, full search is once again applied. Otherwise, the localization is treated as stable (applying Rule-1) and the localization process will move to the next step (applying Rule 2).

Suppose the robot is determined to be stably in a specific venue rather than the corridor, in the next frame the robot can only appear in two venues, i.e. either remaining in the same venue or entering the corridor. As a result, the next test frame is only matched against the training frames from the same venue and the corridor, i.e. a much smaller subset in comparison with the entire training set. This will not only improve the efficiency but also improve both the accuracy and robustness of our approach for robot localization.

If the robot is found stably in the corridor, it will remain in the corridor or move into a new venue. Accordingly, the next frame can be matched with all training frames with the exception of those within previous localized venues. In order to achieve this, the path history of the robot is recorded, and only the last non-corridor venue is excluded in matching for flexibility, i.e. to allow the robot indirectly revisiting a venue.

Considering that both the training and test sequences are captured using the same camera in the same geographic condition, we assume that frames taken in the same location will contain similar contents and consistent geometric information. Thus, our matching process needs to consider this similarity and consistency. The process of matching frames using extracted POIs is discussed in full in the next subsection.

\section{Strategy for Feature Matching}

Inspired by stereo image matching which computes the disparity map of a sequence by estimating the similarity between the left and right channel of image, we consider the training and test sequences as a stereo image sequence in our experiment. Thus, we applied a similar procedure to compute the similarity.

Suppose the matching can be represented by the homography between POIs of an image pair, then POIs mapped from one image to the another image would coincide exactly with their correspondences. Generally, the homography is only an approximation of the mapping, thus mapped POIs cannot coincide exactly, i.e. containing false matches. The acceptance of a putative matching is then to be considered in more detail, this will be discussed in two steps as follows.

\section{1) Local matching to find corresponding POI pairs}

For a given POI in a reference image $I_{1}$, its corresponding POI in image $I_{2}$ is determined via local image matching using Normalized Cross Correlation (NCC) criterion [31]. Let $\left\{x_{i}\right\}, i \in\left[1, N_{1}\right]$ and $\left\{y_{j}\right\}, j \in\left[1, N_{2}\right]$ be two sets of POIs extracted 
from $I_{1}$ and $I_{2}$, respectively. For each $x_{i}$ in $I_{1}$, its best correspondence $y_{j^{*}}$ in $I_{2}$ is determined using NCC as

$$
\begin{gathered}
j^{*}=\underset{k \in\left[1, N_{2}\right]}{\arg \max }\left(\operatorname{NCC}\left(x_{i}, y_{k}\right)\right) \\
N C C\left(x_{i}, y_{j}\right)=\frac{\sum_{W}\left(I_{1}(\tilde{x})-\bar{I}_{1}\right)\left(I_{2}(\tilde{y})-\bar{I}_{2}\right)}{\sqrt{\sum_{W}\left(I_{1}(\tilde{x})-\bar{I}_{1}\right)^{2}\left(I_{2}(\tilde{y})-\bar{I}_{2}\right)^{2}}}
\end{gathered}
$$

where $\tilde{x}$ belongs to $W\left(x_{i}\right)$, a local window in $I_{1}$ centered at $x_{i}$ and $\bar{I}_{1}$ is its mean intensity. Similarly, $\tilde{y} \in W\left(y_{j}\right)$, a local window in $I_{2}$ centered at $y_{j}$ and $\bar{I}_{2}$ is its mean intensity.

If there is another correspondence $\left(i^{\prime}, j^{*}\right)$ determined before, choose $i^{*}$ as the matched point for $j^{*}$ by

$$
i^{*}=\underset{m \in\left\{i, i^{\prime}\right\}}{\arg \max }\left(N C C\left(m, j^{*}\right)\right)
$$

In addition, if the associated NCC value is less than a given threshold $t_{n c c}$, the determined point correspondence is treated as invalid. In our system, $t_{n c c}$ is set as $60 \%$ of the maximum NCC value among all POI correspondences.

\section{2) Global matching using RANSAC}

Once an initial set of POI correspondences are obtained using NCC, the next step is to verify these local correspondences using a global constraint, where homography among these matched pairs of POI is determined to achieve affine-invariant matching of images. The idea behind is based on the fact that images captured from one particular scene in testing and training sets can be regarded as stereo pairs. This is because these images are captured by the same robot under various viewpoints along with possible change of lighting conditions. As a result, these images should share common contents with limited difference that can be tolerated during our homography-based matching. On the other hand, a failed matching may indicate that the test image does not belong to the venue associated to the training one.

To determine the homography between two sets of POI obtained for the test image and the training image, the RANSAC algorithm [14] is used to select an optimized subset of their matched POI correspondences. When the homography is determined, the number of matched POI pairs from the RANSAC algorithm is then denoted as a degree of consistency measurement between the images. Meanwhile, POIs which are excluded in the matching are treated as outliers and disregarded for robustness. According to the POIs extracted from the images in Fig. 5, examples of matched points from two images with different illumination conditions are shown in Fig. 6, where a pair of corresponding POIs is represented by 'green *' and 'red +'. As can be seen in the example, the approach that we have adopted has successfully filtered inconsistent POIs towards reliable matching of images. It is also noteworthy that there are mis-matched points in Fig. 6, and these are mainly due to the fact that the images under processing are not strict stereo pairs. To allow RANSAC to be applied in such a different problem, we adjusted the 
tolerance in the algorithm for robustness. As a result, global matching can still generate satisfactory results despite of these errors in matching.

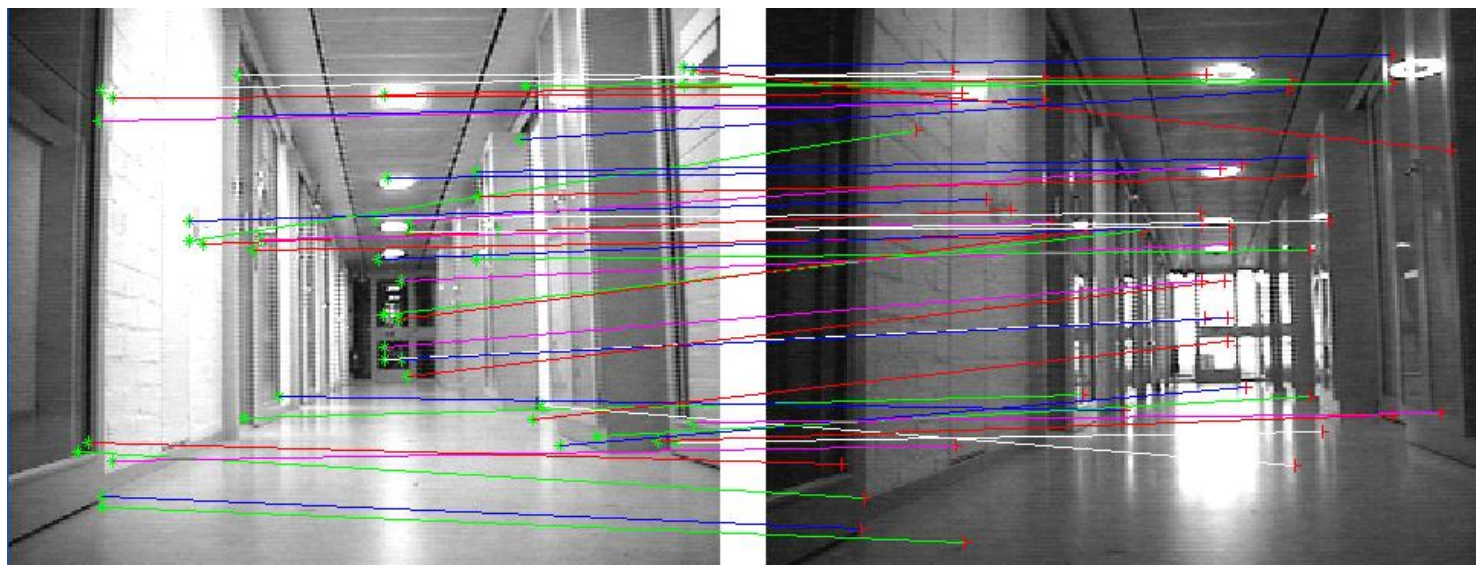

Fig 6. The matching map, the points linked with lines refer to a pair of matched POIs.

When a test image is compared with all frames in the training set, each homography-constrained matching determined by RANSAC generates a measurement of consistency. Rather than specifying the test image into the venue class of the frame who has the highest consistency measurement among all images in the training set, for robustness the first $N_{r}$ best matched frames of highest consistency measurement are obtained to make the final decision. In addition, a diagram of our global matching scheme is presented in Fig. 7 to further explain how it works, and details of which is described below.

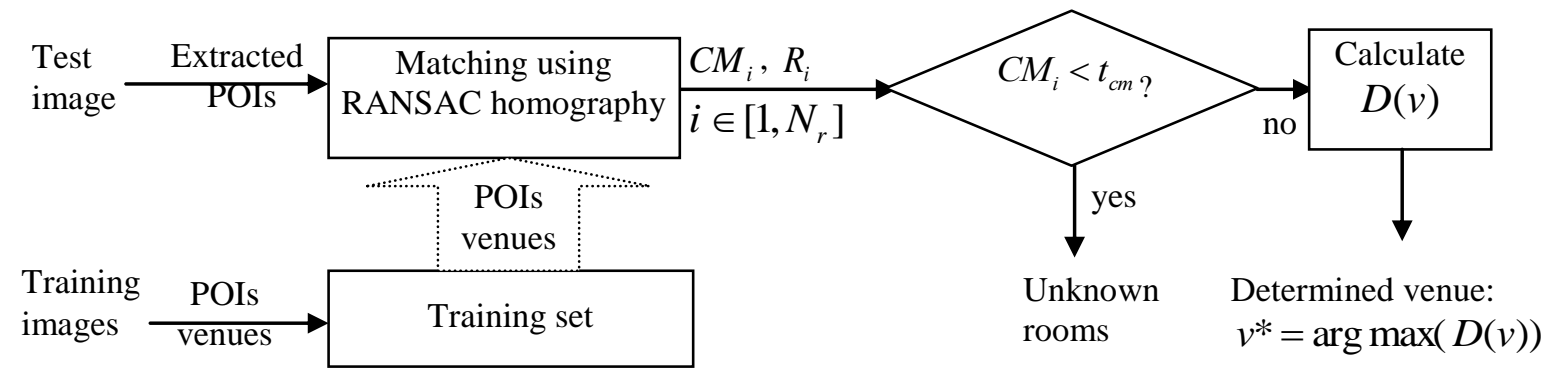

Fig. 7. Block diagram of our global matching scheme.

Let $C M_{i}$ and $R_{i}$ respectively refer to the value of consistency measurement and associated venue of the $i^{\text {th }}$ best match from RANSAC, where $C M_{i} \geq C M_{j}$ if $i<j$ for $i, j \in\left[1, N_{r}\right]$, i.e. $C M_{1}$ is the match of highest consistency measurement. If $C M_{1}$ is less than a given threshold $t_{c m}$, the matching is considered unreliable, which means that the test image has no satisfied matches in the training set. In other words, it is most likely that the test image comes from a venue excluded from the training 
set, i.e. an unknown room. As a result, Rule 3 in Section IV(A) is applied to identify such cases, where $t_{c m}$ is empirically set as

15. Otherwise, the test frame will be associated with one of the five known venues as follows.

Let $v$ be one of the five given venues in the training set, for each of them a matched degree $D($.$) is determined as$

$$
\begin{aligned}
& D(v)=\sum_{i} C M_{i} \cdot \operatorname{Tag}(i, v) \\
& \operatorname{Tag}(i, v)= \begin{cases}1, & \text { if } \quad R_{i}=v \\
0 & \text { otherwise }\end{cases}
\end{aligned}
$$

Then, the test frame is annotated of the venue of maximum matched degree, i.e. $v^{*}=\arg \max (D(v))$. This is consistent with our assumption that the location can be retrieved by finding the most similar frame in the training sequence to the test sequence. As a result, the most similar frame should contain the most number of POIs to match with the test frame.

\section{RESULTS AND EVALUATION}

The proposed approach which has been outlined above was applied for mobile robot localization task in the ImageCLEF workshop in 2009, and the results are summarized and analyzed in this section.

\section{A. Data Set and Evaluation Criteria}

In the robot vision task of ImageCLEF 2009, a subset of KHL-IDOL2 database [27] is used for both training and testing. For each of the three illumination conditions, i.e. cloudy, sunny and night, there are four training sequences which are captured by a camera mounted on a mobile robot. Data from two robots are provided, which form 24 sequences in total. During the testing stage, a single sequence of unknown illumination condition is utilized, which contains new venues which are excluded from the training sequences.

To enable quantitative evaluations, annotated results are introduced in the captured sequences to denote the position of the robot. Such information is available in the training sequences as ground truth maps, and a score is used to measure the accuracy of annotated results in comparison with the ground truth. Actually, the score here attempts to reward systems for correctly annotating frames and also penalizing systems for incorrectly annotating frames, thus giving an overall indication of performance. The following rules are used when calculating the score for a single test sequence:

- $\quad+1.0$ points for each correctly annotated frame.

- Correct detection of an unknown room is treated the same way as correct annotation.

- $\quad-0.5$ points for each incorrectly annotated frame.

- 0 points for each image that was not annotated (the algorithm refrained from the decision). 
In fact, a script is also provided by the ImageCLEF organisers to enable participants to evaluate the performance of their algorithms/systems as this script will generate a score for each annotated sequence by comparing it with the ground truth. According to the obtained scores, all the systems were then ranked and systems of higher scores are considered to be better than those of lower scores.

\section{B. Preliminary Evaluation using Training Sequences}

To measure the performance of our proposed approach over training sequences, two groups of experiments are implemented. In the first group, sequences under the same illumination conditions are used for training and testing. In the second group, on the contrary, sequences of different illumination conditions are mixed together during the training and testing process. Certainly, the first group will generate better results than the second group, but the second group is more practical and indeed a more robust evaluation matching the requirements of ImageCLEF.

In each group of experiments, results of three solutions are compared. The first solution uses only conventional method for detecting POIs, followed by a coarse point matching, and this can be regarded as baseline. The second solution adopts illumination normalized scheme in the extraction of POIs, where the matching scheme remains the same. The third solution is achieved by introducing model-constrained matching into the second solution, thus it can be considered as a much improved version of the baseline system.

Table 1. Performance of three solutions using cross validation

\begin{tabular}{|c|c|c|c|c|c|c|}
\hline \multirow{2}{*}{} & \multicolumn{2}{|c|}{$\begin{array}{c}\text { Solution 1 } \\
\text { (Baseline) }\end{array}$} & \multicolumn{2}{c|}{ Solution 2 } & \multicolumn{2}{c|}{ Solution 3 } \\
\cline { 2 - 7 } & $A_{\text {ccur }}$ & score & $A_{\text {ccur }}$ & score & $A_{\text {ccur }}$ & score \\
\hline Cloudy & $73.1 \%$ & 585.5 & $77.8 \%$ & 640.0 & $79.7 \%$ & 657.5 \\
\hline Sunny & $74.2 \%$ & 600.5 & $78.0 \%$ & 625.0 & $80.2 \%$ & 655.5 \\
\hline Night & $71.9 \%$ & 785.5 & $73.3 \%$ & 815.5 & $76.1 \%$ & 840.5 \\
\hline Mixed & $64.2 \%$ & 565.5 & $71.6 \%$ & 630.5 & $74.8 \%$ & 661.0 \\
\hline
\end{tabular}

Herein, cross validation is employed for performance evaluation, where $75 \%$ of the training sequences are used for training and the remaining $25 \%$ for testing. The average accuracy $A_{c c u r}$ and score obtained are reported in Table 1 . As can be seen, the proposed contrast-based illumination normalization and model-constrained matching indeed can improve the accuracy of the annotation results. In addition it can be seen that such improvements become significant when data of mixed illumination conditions is used. 
Also, it should be noted that a high score may be obtained even the annotation accuracy is low; this can be seen particularly in the results in the third row, i.e. from night conditions. This is brought about because the score and the accuracy are defined in an inconsistent manner. As mentioned above, the score is defined as an accumulated reward or penalty, and the accuracy is an average percentage of correctly annotated frames. Since the reward of 1.0 is higher than 0.5 for penalty, a longer sequence will certainly produce a higher score provided that an average accuracy of more than $50 \%$ is achieved. In fact, the sequences from night conditions are the longest in the whole group, and this explains the results in Table 1. Nevertheless, under a given test sequence such inconsistency can be disregarded, thus the final evaluation achieved is still consistent.

To further analyze the annotated results, a confusion matrix is generated and shown in Table 2. This table provides an indication of how accurate the localization is in terms of the five venues including printer area, corridor, two-person office, oneperson office and kitchen (see for details in Fig. 1 in Section II). Based on the results in Table 2, several observations can be made and summarized as follows. Firstly, due to the similarity between corridor and printer area, about $7.1 \%$ printer area and $11.8 \%$ of corridor frames are mis-annotated as the other. Secondly, the accuracy in annotating two-person office is the highest at nearly $87 \%$ due to its significant difference from other venues. Thirdly, due to two reasons the accuracy for annotating oneperson office is the lowest at about $75 \%$. One is its narrow layout which makes it appear like the corridor. The other is the rectangle-shaped tables, which are very similar to the settings in the kitchen. As a result, $8.1 \%$ and $8.7 \%$ of one-person office frames are mis-annotated as either corridor or kitchen respectively. However, only $3.4 \%$ of corridor frames are mis-annotated as kitchen. Fourthly, about $10.3 \%$ of kitchen frames are annotated as one-person office, this again is due to the similar contents inside these frames. Finally, the average accuracy achieved is around $80.3 \%$ for the five venues, and this shows the effectiveness of our proposed approach.

Table 2. Confusion matrix for the annotation results of five venues including printer area,

corridor, two-person office, one-person office and kitchen.

\begin{tabular}{|c|c|c|c|c|c|}
\hline & Printer area & Corridor & Two-person & One-person & Kitchen \\
\hline Printer area & $82.2 \%$ & $7.1 \%$ & $2.9 \%$ & $3.3 \%$ & $4.5 \%$ \\
\hline Corridor & $11.8 \%$ & $80.0 \%$ & $2.7 \%$ & $2.1 \%$ & $3.4 \%$ \\
\hline Two-person & $2.8 \%$ & $4.5 \%$ & $86.9 \%$ & $2.5 \%$ & $3.3 \%$ \\
\hline One-person & $6.1 \%$ & $8.1 \%$ & $1.9 \%$ & $75.2 \%$ & $8.7 \%$ \\
\hline Kitchen & $4.8 \%$ & $2.6 \%$ & $4.2 \%$ & $10.3 \%$ & $78.1 \%$ \\
\hline
\end{tabular}

To further evaluate the performance of our proposed method, cross validation using 12 sequences provided by ImageCLEF 2009 is carried out. Basically, these sequences are captured under three lighting conditions, i.e. cloudy, night, and sunny. 
Different combinations of these sequences form 12 tests and the results are report in Table 3 . As can be seen, an average score of 619.12 is obtained, which corresponds to an average accuracy of $78.2 \%$. Also please note that under same accuracy, the scores can be significantly different, see for example for the tests in groups $\mathrm{H}$ and $\mathrm{K}$. To overcome such inconsistency in evaluation, we introduce a normalized score $\hat{S}$ defined as $\hat{S}=S_{\text {raw }} / l$, where $S_{\text {raw }}$ is the raw score obtained using previous evaluations and $l$ refers to the number of frames in the test sequence. Accordingly, normalized score for each test is calculated and also compared in Table 3. As seen, for test groups $\mathrm{H}$ and $\mathrm{K}$, their normalized scores are quite similar to each other, thus the proposed normalized score $\hat{S}$ provides a more consistent measurement of performance with the obtained accuracy.

Table 3. Results of cross validation to further validate the effectiveness of proposed method.

\begin{tabular}{|c|c|c|c|c|c|c|}
\hline \multirow[b]{2}{*}{ Tests } & \multirow[b]{2}{*}{ Test seq } & \multirow{2}{*}{$\begin{array}{c}\text { Frames } \\
(l)\end{array}$} & \multirow[b]{2}{*}{ Training seq } & \multirow[b]{2}{*}{$A_{c c u r}$} & \multicolumn{2}{|c|}{ Score } \\
\hline & & & & & $S_{\text {raw }}$ & $\hat{S}$ \\
\hline \multirow{3}{*}{ A } & \multirow{3}{*}{ Cloudy_1 } & \multirow{3}{*}{917} & Cloudy_2 & \multirow{3}{*}{$87.9 \%$} & \multirow{3}{*}{745} & \multirow{3}{*}{0.8124} \\
\hline & & & Night_1 & & & \\
\hline & & & Sunny_1 & & & \\
\hline \multirow{3}{*}{ B } & \multirow{3}{*}{ Cloudy_2 } & \multirow{3}{*}{928} & Cloudy_3 & \multirow{3}{*}{$75.4 \%$} & \multirow{3}{*}{639} & \multirow{3}{*}{0.6886} \\
\hline & & & Night_2 & & & \\
\hline & & & Sunny_2 & & & \\
\hline \multirow{3}{*}{$\mathrm{C}$} & \multirow{3}{*}{ Cloudy_3 } & \multirow{3}{*}{915} & Cloudy_4 & \multirow{3}{*}{$78.4 \%$} & \multirow{3}{*}{625} & \multirow{3}{*}{0.6831} \\
\hline & & & Night_3 & & & \\
\hline & & & Sunny_3 & & & \\
\hline \multirow{3}{*}{$\mathrm{D}$} & \multirow{3}{*}{ Cloudy_4 } & & Cloudy_1 & & & \\
\hline & & 1022 & Night_4 & $73.0 \%$ & 600 & 0.5871 \\
\hline & & & Sunny_4 & & & \\
\hline & & & Cloudy_1 & & & \\
\hline $\mathrm{E}$ & Night_1 & 965 & Night_2 & $84.5 \%$ & 740 & 0.7668 \\
\hline & & & Sunny_1 & & & \\
\hline & & & Cloudy_2 & & & \\
\hline $\mathrm{F}$ & Night_2 & 951 & Night_3 & $73.6 \%$ & 619 & 0.6509 \\
\hline & & & Sunny_2 & & & \\
\hline & & & Cloudy_3 & & & \\
\hline G & Night_3 & 1034 & Night_4 & $68.4 \%$ & 542.5 & 0.5247 \\
\hline & & & Sunny_3 & & & \\
\hline & & & Cloudy_4 & & & \\
\hline $\mathrm{H}$ & Night_4 & 954 & Night_1 & $77.9 \%$ & 635 & 0.6656 \\
\hline & & & Sunny_4 & & & \\
\hline & & & Cloudy_1 & & & \\
\hline I & Sunny_1 & 893 & Night_1 & $88.4 \%$ & 747.5 & 0.8371 \\
\hline & & & Sunny_2 & & & \\
\hline & & & Cloudy_2 & & & \\
\hline $\mathrm{J}$ & Sunny_2 & 908 & Night_2 & $80.2 \%$ & 626 & 0.6894 \\
\hline & & & Sunny_3 & & & \\
\hline & & & Cloudy_3 & & & \\
\hline $\mathrm{K}$ & Sunny_3 & 485 & Night_3 & $77.4 \%$ & 320.5 & 0.6608 \\
\hline & & & Sunny_4 & & & \\
\hline & & & Cloudy_4 & & & \\
\hline $\mathrm{L}$ & Sunny_4 & 999 & Night_4 & $72.9 \%$ & 590 & 0.5906 \\
\hline & & & Sunny_1 & & & \\
\hline Avg. & & 914.25 & & $78.2 \%$ & 619.12 & 0.6798 \\
\hline
\end{tabular}


To illustrate the similarity between frames from different venues, Fig. 8 gives six typical frames extracted from the training set, which form three pairs shown in three columns. As can be seen, each pair of images is of quite similar content and layout but annotated differently. In other words, this shows the difficulty in venue determination for automatically robot localization using vision only information. As a result, the relative high accuracy achieved appears reasonably good.
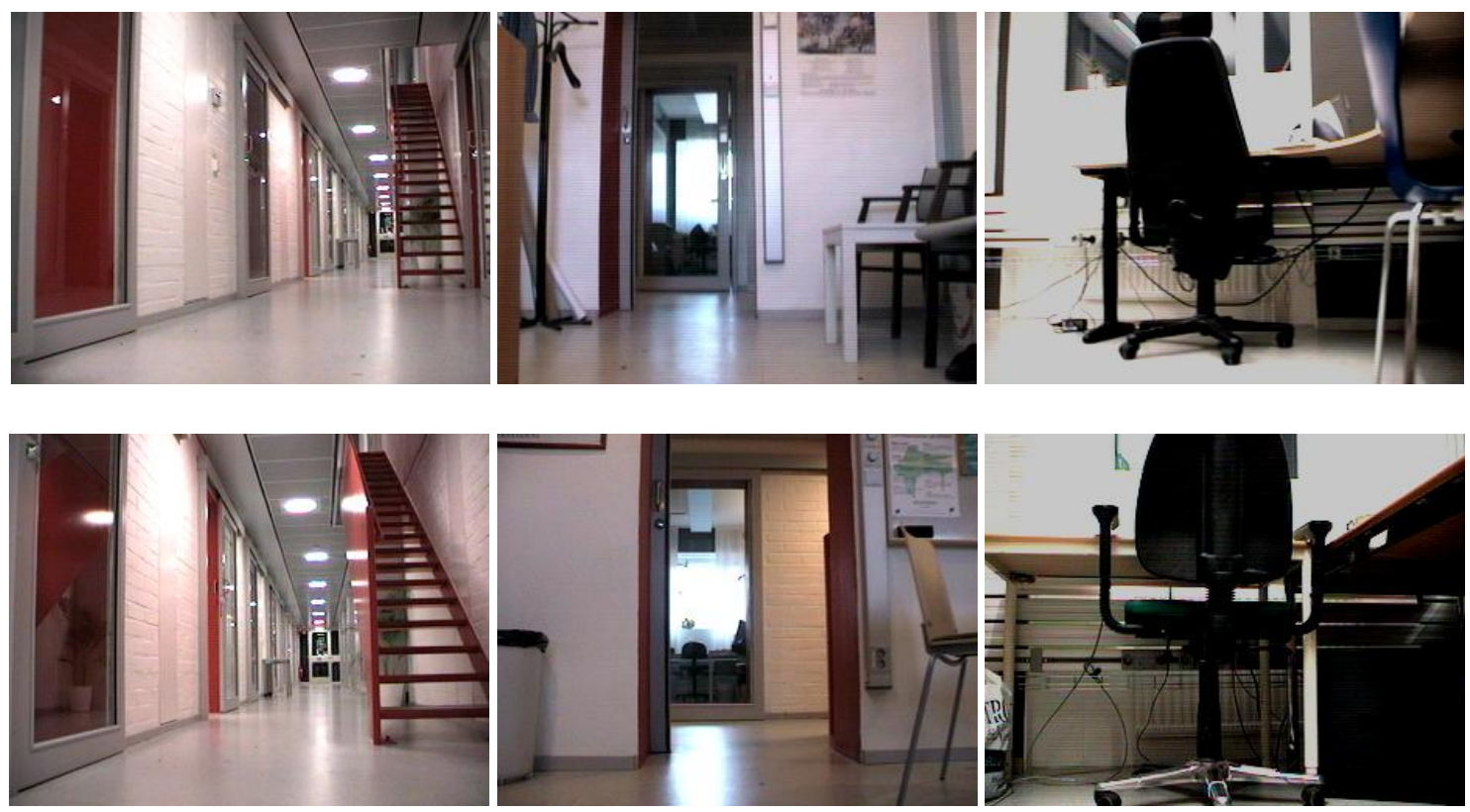

Fig 8. Examples of typical frames to illustrate the similarity of frames from different venues: The left column is from printer area and corridor; the middle column is from two-person office and kitchen; and the last column is from two-person office and one-person office, respectively.

\section{Performance of ImageCLEF Official Evaluation}

As mentioned previously, in the official evaluation only one sequence with unknown illumination condition and newly introduced venues was utilized. Based to the results announced by ImageCLEF 2009, the scores for the first four teams/systems are summarized in Table 4, where our proposed system is ranked as being second in the optional task group [29]. As can be seen, the best results achieved by the first three teams are quite comparable with a score around 900, for all of these approaches this is mainly due to the usage of neighboring frames in the past. Without utilizing this neighboring information, as reported in another task group, the highest score achieved is only 793.0 by the IDIAP team [19].

Table 4. Results of the best 4 systems and their scores [19, 32]

\begin{tabular}{|c|c|c|c|c|}
\hline Team/System & SIMD & Proposed & CVIU & IDIAP \\
\hline Score & 916.5 & 890.5 & 884.5 & 853.0 \\
\hline
\end{tabular}


It is worth noting that using neighboring frames after the current frame may significant improve the accuracy. For example, in a system proposed by CVIU the label of a test frame is iteratively corrected to the most frequent label in the sequence of \pm 20 frames until convergence. Using such a smoothing procedure, a score of more than 1000 can be achieved. However, this is considered based on the rules of the robot localization task as invalid because the annotation relies on future information after the frame which is being annotated, which means that it is almost impossible to make a real time decision about the current location.

In the following, techniques used by the other three teams listed in Table 4 are discussed and compared. In the SIMD system [33], a Monte-Carlo particle-filter is used to track the robot pose with extracted scale-invariant feature transform (SIFT) features to update the tracker. The pose $(x, y, \theta)$ includes 2D position $(x, y)$ and an orientation $\theta$ of the robot. Despite the high complexity, this solution seems to lack flexibility, as the training process also relies on information provided by a distance sensor rather than only the camera output. In the CVIU system, a Gaussian gradient on the L component of the LAB color space is utilized as the main feature together with histograms from a three-tie spatial pyramid. Then a SVM is employed to compute the probability of a test frame to each room. Finally, a smoothing procedure is applied as post-processing by exploring multiple images, i.e. the label of a test frame is corrected to the most frequent label in the sequence of the past 20 frames. Although the smoothing procedure seems to satisfy the requirements of ImageCLEF 2009, details on how to avoid mis-annotation when the robot moves from one location to another are absent as this appears to be corrected via smoothing. In the IDIAP system, the main techniques used include Composed Receptive Fields Histograms (CRFH) and SIFT features in combination with a HarrisLaplace detector, a Generalized Discriminative Accumulation Scheme (G-DAS) and SVM classifier, and relevant details can be found in $[15,28]$. Temporal filtering for smoothing the results is also applied using five images before the classified image.

In summary, point based features like SIFT and POI detector are widely used in this task, including the system from SIMD, IDIAP and us. Without complex features, tracking and learnt classifiers, our proposed method has yielded a reasonably good performance. Further analysis of the errors in annotation is discussed in the next section.

\section{Analysis and Discussions}

Using the available information, it is interesting to compare the annotated results from our system and SIMD with the published ground truth maps, and the results are shown in Table 5. In 1690 frames of the test sequence, our approach can correctly annotate 1157 frames with an accuracy of about $68.5 \%$. On the contrary, the accuracy of SIMD is only $63.8 \%$ if the rejected frames are considered as incorrect. However, SIMD has gained a higher score than our system because of the penalty caused by many more incorrectly classified frames in our system. Again, this shows the inconsistency of evaluation using the accuracy and score measurements. 
In 533 misclassified frames, 227 or $42.6 \%$ of them are from unknown rooms, i.e. absent from training sequences. In total 121 frames are classified as being from unknown rooms in our system, of which 114 or $94.2 \%$ frames are correct. In other words, we have a low recall rate but a pretty high precision rate in detecting unknown rooms.

Table 5. Detailed results from SIMD and our system

\begin{tabular}{|c|c|c|c|c|}
\hline Team/System & Correct & Wrong & Reject & Accuracy \\
\hline SIMD & 1072 & 311 & 297 & $63.8 \%$ \\
\hline Our & 1157 & 533 & 0 & $68.5 \%$ \\
\hline
\end{tabular}

When analyzing the errors in our system, it is found that most of the incorrectly annotated frames are those transitional frames, i.e. when the robot leaves one venue or enters another. There are two main difficulties in addressing these transitional frames. In the first case, as shown in Fig. 9, frames taken close to the door (while the robot is entering a room) are very often lacking in texture, which makes extraction and matching of POIs impractical. More importantly, in the second case, these transitional frames may share similar visual contents but are associated with different venue tags, i.e. potential ambiguity in ground truth maps (see, for example, the images in the left column of Fig. 8).
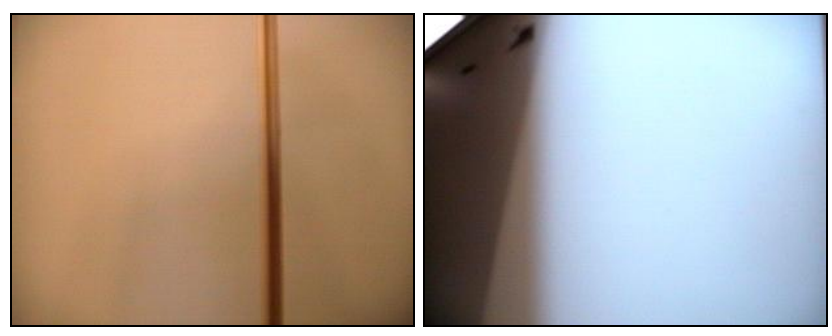

Fig 9. Example frames with limited texture information for POI extraction.

To explain such ambiguity, it is necessary to discuss how these ground truth maps are obtained. In fact, the robot pose is estimated during the acquisition process using a laser based localization method. Each image is labeled as belonging to one of the five venues based on the position from where it was taken. As a result, images taken, for example, from the corridor, but looking into a room are labeled as corridor, even though their visual contents are those from inside a given venue [15, 28].

From the point of view of image matching, it is very hard to make an appreciate decision in the two cases outlined above using the image content only, especially when feedback from future frames is absent. On the other hand, it might be useful to reject making decisions in these cases, and this certainly will improve the score that our approach achieved by avoiding penalty caused by misclassification. 
Regarding computational performance, overall our system needs 1.36 s to test each frame, in which most of the time (over 90\%) is spent for POI extraction and matching. This performance is decent under implementation using Matlab, in comparison to SIMD system, which requires over 4 s to process each test image. When the system was converted to $\mathrm{C} / \mathrm{C}++$ implementation with multi-thread based parallel processing, it only requires $0.18 \mathrm{~s}$ to process one frame when it is tested on a quad-core CPU with 4GB RAM. Further improvements in speed can be achieved by introducing hardware accelerator and strategies like skipping frame as used in other systems [39].

\section{E. Analysis of Important Parameters}

In this subsection, the effects of several important parameters in the proposed method are discussed. These include $t_{n c c}$ and $t_{c m}$ which were introduced in Section IV, which respectively refer thresholds to filter unreliable point pairs in NCC and to detect unknown frames in RANSAC. In fact, in our system these parameters are empirically decided during the training process by maximizing the obtained classification scores. Please note the parameters determined are not guaranteed to be global optimal, this is simply because the test case containing unknown frames is different from the training one. However, it still provides a practical solution for this task.

Regarding $t_{n c c}$, it refers to a percentage of the highest matching value during NCC in generating corresponding POI pairs.

Larger $t_{n c c}$ will lead to less validated POI pairs, thus it will speed up the matching of RANSAC, and visa versa. On the other hand, too few POI pairs may cause unreliable estimation of the homography by RANSAC. As a result, a suitable value of $t_{n c c}$ should be selected as a trade-off between the robustness and the efficiency of the estimate. To achieve this, we repeat the experiments in Section $\mathrm{V}(\mathrm{B})$ with various values of $t_{n c c}$ and the results are given in Table 6. As can be seen in Table 6, $t_{n c c}=0.6$ generates the highest score in this test and thus the threshold is determined as 0.6.

Table 6. Overall testing scores vs. various $t_{n c c}$ values.

\begin{tabular}{|c|c|c|c|c|c|c|}
\hline$t_{n c c}$ & 0.45 & 0.5 & 0.55 & 0.6 & 0.65 & 0.70 \\
\hline Score & 624.0 & 636.5 & 650.0 & 661.5 & 647.5 & 627.0 \\
\hline
\end{tabular}

To determine $t_{c m}$ in detecting unknown rooms in the testing sequence, we need to simulate unknown rooms in the training set, as these are absent from the training sequences. Accordingly, we respectively treat three venues including kitchen, oneperson office and two-person office as unknown rooms. Consequently, the corresponding frames are excluded in training but 
used for testing under similar settings as outlined above. The average testing score from three manually introduced unknown cases is then obtained, as shown in Table 7. As can be seen, the highest score is generated by setting $t_{c m}=15$.

Table 7. Overall test scores vs. various $t_{c m}$ values.

\begin{tabular}{|c|c|c|c|c|c|}
\hline$t_{c m}$ & 5 & 10 & 15 & 20 & 25 \\
\hline Score & 632.0 & 657.5 & 682.0 & 668.5 & 643.5 \\
\hline
\end{tabular}

\section{CONCLUSIONS}

In this paper we have described a vision-based framework for mobile robot localization and applied our approach as part of the robot vision task for ImageCLEF 2009. The outlined approach is applicable to indoor environments to classify the current location of the robot into several predefined venues including unknown venues. The main contributions of this paper can be summarized as follows.

- A novel POI image matching algorithm with consideration for the contrast effect has been developed. A certain amount of performance improvement is gained by using POI instead of using all of the pixels in the frame. Since the main assumption for the whole system has been that edge corner points contain more valuable information than other pixels, the reduced number of pixels for image matching results in a higher accuracy and overall efficiency for the overall system. The normalized contrast has the goal of keeping the effect from the lighting conditions to a minimum so that the system performance achieved can be as good as possible.

- Modeling of robot behavior for model-constrained matching has been designed to simulate the moving behavior of the robot. This approach is aiming to refine the results from image matching using context constraints. Such constraints may predict and limit the possible annotated results, thus the exhaustive matching process is optimized for improved efficiency, accuracy, and robustness.

- A normalized score is proposed for consistent performance evaluation of these systems.

The evaluated results show our proposed method achieved the second highest score in the robot vision task in ImageCLEF 2009, ranked just below the SIMD system. However, unlike the SIMD system, our approach did not rely on data from other sensors such as spatial location of the robot for training, thus it provided a more general and flexible vision-based framework for the robot vision task. The experimental results outlined in this paper show that model constrained matching is successful for robot localization since it considers visual information along with the motion behavior of the mobile robot. The results also 
reflect the magnitude of the difficulty of the problem at robot vision, such as how to annotate an unknown room correctly for example. While we have gained much insight into the practical problems involved in robot localization, future work will be focusing on how to accurately detect unknown rooms and deal with transitional frames. With further improved modeling of the two cases above, including possible rejected decision-making in dealing with transitional frames, we believe that much better results can be produced using our proposed methodology.

\section{REFERENCES}

[1] ImageCLEF (Robot vision task), http://www.imageclef.org/2009/robot

[2] G. N. Desouza, A. C. Kak: "Vision for mobile robot navigation: a survey," , IEEE Trans. Pattern Analysis and Machine Intelligence, vol.24, no.2, pp.237267, Feb 2002 doi: 10.1109/34.982903

[3] J. Kim and H. Jun: "Vision-based location positioning using augmented reality for indoor navigation," IEEE Trans. Consumer Electronics, vol. 54, no. 3, pp. 954-962, Aug. 2008, doi: 10.1109/TCE.2008.4637573

[4] M. A. Rubin, W. T. Freeman, K. P. Murphy, and A. Torralba: "Context-Based Vision System for Place and Object Recognition," in Proc. Int'l Conf. Computer Vision, vol. 1, pp. 273-280, 2003

[5] A. Oliva and A. Torralba: "Modeling the Shape of the Scene: a Holistic Representation of the Spatial Envelope," Int'J in Computer Vision, vol. 42, pp. 145-175, 2001.

[6] I. Ohya, A. Kosaka, and A. Kak: "Vision-based navigation by a mobile robot with obstacle avoidance using single-camera vision and ultrasonic sensing," , IEEE Trans. Robotics and Automation, vol.14, no.6, pp.969-978, Dec 1998

[7] M. Hashima, F. Hasegawa, S. Kanda, T. Maruyama, and T. Uchiyama: "Localization and obstacle detection for robots for carrying food trays," in Proc. the IEEE/RSJ Int. Conf. Intelligent Robots and Systems (IROS '97), vol.1, pp.345-351, 7-11 Sep 1997

[8] P. Althaus and H.I. Christensen. 'Behavior coordination in structured environments.' Advanced Robotics, vol. 17, no. 7, pp. 657-674, 2003

[9] A. Pronobis and B. Caputo: "Confidence-based cue integration for visual place recognition," in Proc. the IEEE/RSJ Int. Conf. Intelligent Robots and Systems (IROS'07), pp. 2394-2401, Oct. 29-Nov. 2, 2007

[10] C. Harris, M. Stephens, “A combined corner and edge detector,” in Proc. 4th Alvey Vision Conference, pp. 147-152, 1988.

[11] H. P. Moravec. “Visual Mapping by a Robot Rover,” Int. Joint Conf. Artificial Intelligence, pp. 598-600, 1979.

[12] M. Gevrekci, B. K. Gunturk, "Illumination robust interest point detection,” Computer Vision and Image Understanding, vol. 113, pp. 565-571, 2009

[13] A. Michelson: Studies in Optics. U. of Chicago Press, 1927.

[14] L. Lu, X. Dai and G. Hager, "Efficient particle filtering using RANSAC with application to 3D face tracking", Image and Vision Computing, Vol. 24, No. 6, pp. 581- 592, June 2006.

[15] A. Pronobis, O. M. Mozos, and B. Caputo: "SVM-based discriminative accumulation scheme for place recognition,” Proc. the IEEE Int. Conf. Robotics and Automation, pp. 522-529, Pasadena, USA, 2008.

[16] J. Liu, M. Wan and J. Zhang, "Monocular robot navigation using invariant natural features," in Proc. 7th World Congress on Intelligent Control and Automation (WCICA'08), pp.5733-5738, Chongqing, China, June 2008

[17] J. Yen and N. Pfluger, "A fuzzy logic based extension to Payton and Rosenblatt's command fusion method for mobile robot navigation," IEEE Trans. Sys. Man and Cybernetics, vol. 25, no. 6, pp. 971-78, 1995 
[18] R. Cipolla and P. J. Giblin, Visual Motion of Curves and Surfaces. Cambridge, U.K.: Cambridge Univ. Press, 2000.

[19] A. Pronobis, L. Xing, and B. Caputo, “Overview of the CLEF 2009 robot vision track,” Lecture Notes in Computer Science, vol. 6242, pp. 110-119, 2010.

[20] J. A. Castellanos, J.M. Martinez, J. Neira, and J.D. Tardos, "Simultaneous map building and localization for mobile robots: a multisensor fusion approach," in Proc. IEEE Int. Conf. Robotics and Automation, vol.2, pp.1244-1249, 16-20 May 1998

[21] B. Yamauchi, A. Schultz and W. Adams, "Mobile robot exploration and map-building with continuous localization", in Proc. IEEE Int. Conf. Robotics and Automation, vol.4, pp.3715-3720, 16-20 May 1998

[22] R. Sim and G. Dudek, "Mobile robot localization from learned landmarks," in Proc. IEEE/RSJ Int. Conf. Intelligent Robots and Systems, vol.2, pp.10601065, 13-17 Oct 1998

[23] S. Se, D. Lowe, and J. Little, "Mobile robot localization and mapping with uncertainty using scale-invariant visual landmarks", Int. Journal of Robotics Research, vol. 21, pp. 735-758, 2002.

[24] D. G. Lowe, “Object recognition from local scale-invariant features”, in Proc. $7^{\text {th }}$ IEEE Int. Conf. Computer Vision, vol. 2, pp. 1150-1157, 1999

[25] L. Wang, H. Li and R. Hartley, "Video Local Pattern based Image Matching for Visual Mapping," in Proc. 18 ${ }^{\text {th }}$ Int. Conf. Pattern Recognition (ICPR), vol. 3, pp. 67-70, 2006

[26] H. Katsura, J. Miura, M. Hild, and Y. Shirai, "A view-based outdoor navigation using object recognition robust to changes of weather and seasons," in Proc. IEEE/RSJ Int. Conf. Intelligent Robots and Systems (IROS'03), vol. 3, pp. 2974-2979, 27-31 Oct. 2003

[27] J. Luo, A. Pronobis, B. Caputo, and P. Jensfelt: "Incremental learning for place recognition in dynamic environments," in Proc. IEEE/RSJ Int. Conf. Intelligent Robots and Systems (IROS'07), pp. 721-728, San Diego, USA, Oct. 2007

[28] A. Pronobis, B. Caputo, P. Jensfelt, and H. I. Christensen: “A realistic benchmark for visual indoor place recognition,” Robotics and Autonomous Systems, vol. 58 , no. 1 , pp. $81-96,2010$

[29] Y. Feng, M. Halvey, and J. M. Jose, “University of Glasgow at Image CLEF 2009 robot vision task,” LNCS, vol. 6242, pp. 295-298, 2010

[30] M. Pavel, G. Sperling, T. Riedl, and A. Vanderbeek, "Limits of visual communication: the effect of signal-to-noise ratio on the intelligibility of American Sign Language,” J. of Optical Society of America (JOSA), vol. 4, no. 12, pp. 2355-2365, 1987.

[31] K. Briechle and U. D. Hanebeck, “Template matching using fast normalized cross correlation,” in Proc. SPIE (Optical Pattern Recognition XII), vol. 4387, pp. 95-102, March 2001.

[32] J. Martinez-Gomez, A. Jimenez-Picazo, and I. Garcia-Varea, "A particle-filter-based self-localization method using invariant features as visual information," in Proc. CLEF workshop, Corfu, Greece, 2009 (SIMD)

[33] A. Pronobis, O. Martínez Mozos, and B. Caputo, "SVM-based discriminative accumulation scheme for place recognition," In Proc. IEEE Int. Conf. Robotics and Automation (ICRA'08), Pasadena, CA, USA, May 2008 (IDIAP)

[34] Y. Gao and Y. Li, "Topological localization of mobile robots using probabilistic support vector classification," Lecture Notes in Computer Science, vol. 6242, pp. 255-260, 2010 (CVIU)

[35] A.-M. Zou, Z.-G. Hou, S.-H. Fu and M. Tan, "Neural networks for mobile robot navigation: a survey,” Lecture Notes in Computer Science, vol. 3972, pp. 1218-1226, 2006.

[36] D. Filliat and J.-A. Meyer, "map-based navigation in mobile robots. I. a review of localization strategies," Cognitive Systems Research, vol. 4, no. 4, pp. 243-282, Dec. 2003.

[37] D. G. Lowe, "Distinctive image features from scale-invariant keypoints," Int. J. Computer Vision (IJCV), vol. 60, no. 2, pp. 91-110, 2004

[38] C. Schmid, R. Mohr, and C. Bauckhage, "Evaluation of interest point detectors,” Int. J. Computer Vision (IJCV), vol.37, no. 2, pp. 151-172, June 2000 
[39] P. Pedreiras, F. Teixeira and N. Ferreira et al, "Enhancing the reactivity of the vision subsystem in autonomous mobile robots using real-time techniques," RoboCup Symposium: Papers and Team Description Papers; also in I. Noda, A. Jacoff, A. Bredenfeld, and Y. Takahashi, editors, RoboCup-2005: Robot Soccer World Cup IX, LNAI, Springer, 2006 